\section{Direct Measurement of Supersonic Skin Friction}

Donald Coles

Research Engineer, Jet Propulsion Laboratory, California Institute of Technology, Pasadena, Calif.

July 2, 1952

$\mathbf{R}$ ECENT EXPERIMENTS AT THE Jet Propulsion Laboratory have established the effect of compressibility on local skin friction for Mach Numbers up to 4.5. Friction coefficients were obtained simultaneously at three stations on a flat plate, for both laminar and turbulent boundary-layer flow, for Reynolds Numbers from $4 \times 10^{5}$ to $1 \times 10^{7}$. The floating element technique used is similar to that of Liepmann and Dhawan. ${ }^{3}$

Figs. 1 and 2 show typical measurements with a clean leading edge and with a tripping device. The Reynolds Numbers in the figures are based on the distance from the leading edge and on the free-stream velocity and kinematic viscosity. No corrections for local pressure gradient or Mach Number variation have been made.

Fig. 3 shows the dependence of turbulent local friction on Mach Number at a Reynolds Number of $8 \times 10^{6}$. It is believed that these data will remain substantially unchanged by any corrections to be applied during the analysis of the experiments.

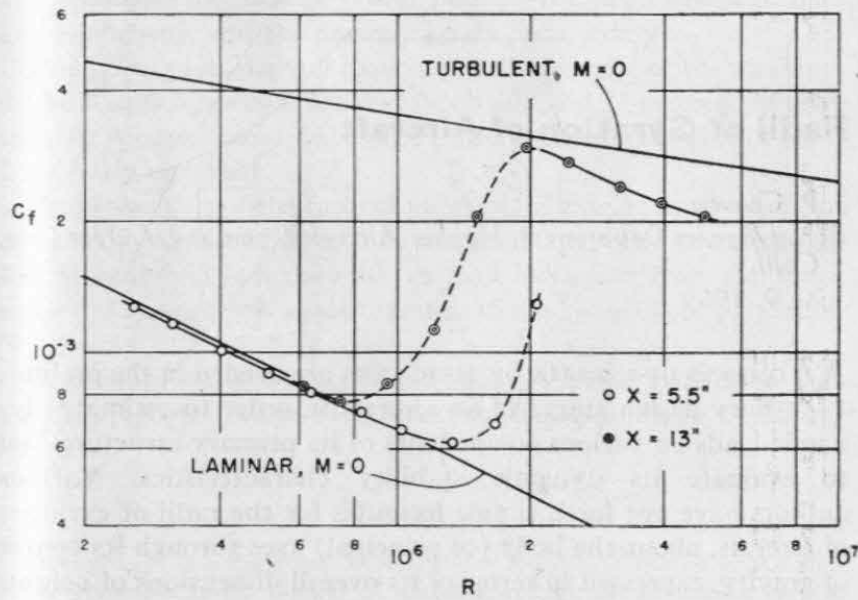

F IG. 1. Local friction on a smooth flat plate at a Mach Number of 2.6 ; no tripping device.

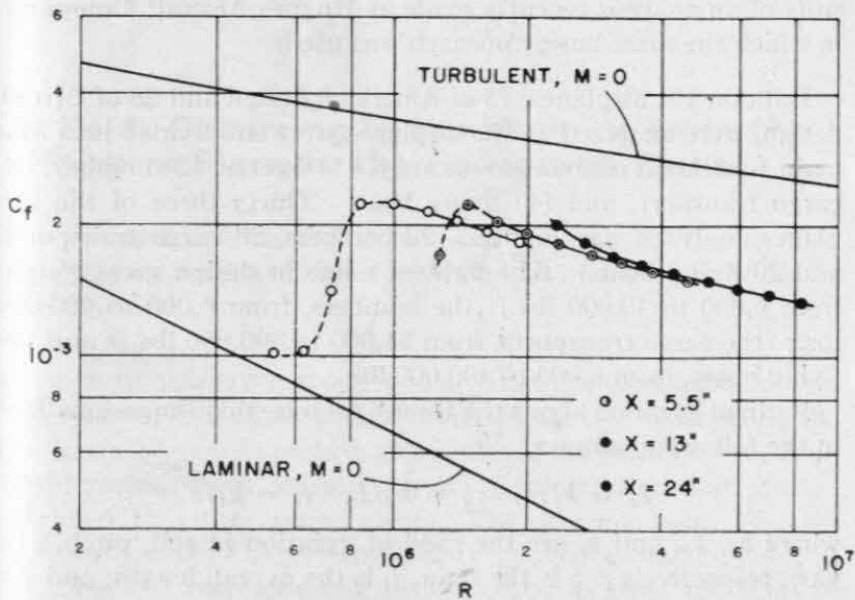

FIG. 2. Local friction on a smooth flat plate at a Mach Number of 3.7 with trìpping device.

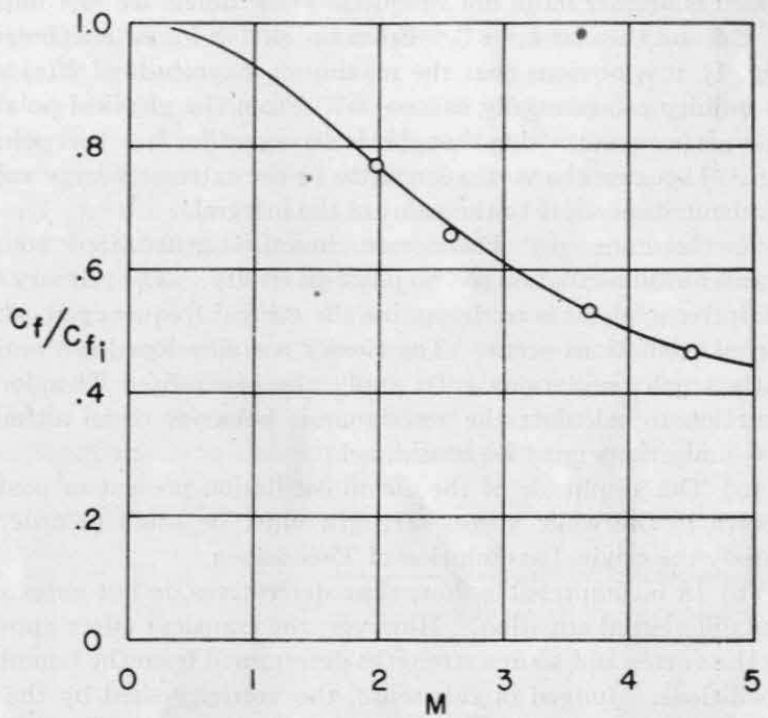

Fig, 3. Variation of turbulent local skin friction coefficient with Mach Number at a Reynolds Number of $8,000,000$.

The boundary-layer research summarized here was discussed in detail at the Annual Summer Meeting of the Institute of the Aeronautical Sciences in Los Angeles in July, 1952.

\section{REFERENCE}

1 Liepmann, H., and Dhawan, S., Direct Measurements of Local Skin Friction in Low-Speed and High-Speed Flow, Proc., 1st U. S. National Congress of Applied Mechanics, Illinois Institute of Technology, 1951; see also Dhawan, S., Direct Measurements of Skin Friction, N.A.C.A. T.N. No. 2567 , January, 1952 .

\section{On Theodorsen Function in Incompressible Flow and C-Function in Supersonic Flow}

\section{Chieh-Chien Chang}

Research Contract Director, The Johns Hopkins University, and Consultant, Air Research and Development Command, U.S.A.F. June 6, 1952

Four interesting PaPers ${ }^{1-4}$ in the Reader's Forum of the March Journal discuss the validity of generalizing the Theodorsen function to the stable airfoil oscillation. To readers not too familiar with flutter problems, it should be emphasized that these discussions are not on the validity of the original Theodor$\operatorname{sen}^{5}$ function. The current confusion lies entirely in the extension of the Theodorsen function to stable oscillations.

In the notation of reference $1, k=k_{0}+i k_{1}$ (where $k_{0}$ is real, positive and $k_{1}$ is real). The references ${ }^{1-4}$ take $k_{1} \lesseqgtr 0$, respectively for unstable, harmonic, and stable oscillations. A sketch shows airfoils in flight at constant velocity $-V$, leaving wake vortex sheets corresponding to the three cases, $k_{1} \lesseqgtr 0$. It is assumed that all the airfoils started from rest at past time $-s / V$ and produced wakes of equal finite length $s$. Observe that the maximum magnitude of vortex strength $U(s)^{*}$ in the wake sheet exponentially decreases with $s$ for $k_{1}<0$, is constant for $k_{1}=0$, and exponentially increases with $s$ for $k_{1}>0$. The limit of case (b) as $s \rightarrow \infty$ is the Theodorsen function $c(k)$. Actually,

$$
c(k)=\int_{1}^{\infty} \frac{s}{\sqrt{s^{2}-1}} e^{i k s} d s / \int_{1}^{\infty} \frac{s+1}{\sqrt{s^{2}-1}} e^{i k s} d s
$$

* The author is fully aware that for a finite wake the vortex strength also depends on the time. $U(s)$ is purely for demonstration. 